\title{
Measuring Electronic Spin-Precession in Magnetic Thin Films by Spin-Polarized Low-Energy Electron Microscopy
}

\author{
C. Klein* and A.K. Schmid* \\ * National Center for Electron Microscopy, Lawrence Berkeley National Laboratory, Berkeley, CA \\ 94720
}

Spin-precession of hot electrons during ballistic propagation through single-crystalline magnetic thin films was studied by spin-polarized low-energy electron microscopy (SPLEEM). Using SPLEEM it is possible to directly observe a spin-dependent interference pattern produced by superposition of the two branches of an electron beam which are created by partial reflection at the film surface and partial reflection at the film-substrate interface. This electronic analog of an optical Fabry-Perot interferometer shows reflectivity maxima for certain electron energies, revealing the existence of quantum well states. The electron energies necessary for the formation of quantum interference maxima and minima are directly related to thickness and band structure of a given film and are therefore spin-dependent for magnetic materials. These effects can not only be utilized to determine the spin-resolved unoccupied electronic band structure of magnetic materials [1] but also to study the influence of magnetic thin films on the polarization vector of an arbitrarily polarized electron beam as demonstrated in the present study.

In our experiments ultra-thin magnetic films were grown on W(110) single crystals by evaporation from a water-cooled electron beam evaporator. The growth temperature was optimized in order to promote the formation of several micron large, atomically flat islands, which cover multiple terraces of the underlying substrate (Fig. 1a). By observing the characteristic occurrence of quantum well states in energy-dependent electron reflectivity measurements ranging from $0 \mathrm{eV}$ to $22 \mathrm{eV}$ above vacuum level we were able to determine the exact local film thickness. The energy- and thicknessdependent reflectivity obtained by such energy scans was evaluated for spin-polarization of the incident electron beam parallel and anti-parallel to the magnetization of the film as well as for perpendicular orientation (Fig. 1b). The latter result is exactly the arithmetic average of the former two, consistent with what is expected for independent interference of spin-up and spin-down parts of an electron wave function. For this reason, information on electron precession can be extracted from measurements with parallel and anti-parallel polarization alone, even though no precession occurs in these two cases. As suggested by Oberli et al. [2], the precession angle in our experiment is equal to the energy-dependent relative phase-shift between spin-up and spin-down part of an electron wavefunction. We evaluated this phase-shift at fixed electron energy for various film thicknesses and found almost perfect linear dependence. By measuring the slope of the corresponding linear regression line we can determine the precession angle per atomic layer (Fig. 1c). This procedure removes all interface effects, which might influence the absolute value of the precession angle, and results in a very accurate determination of a basic property of magnetic materials, i.e. the energydependent electronic spin-precession angle per atomic layer.

As shown by Weber et al. [3] spin-precession of electrons in magnetic films is one crucial mechanism in connection with the development of the technique of magnetization reversal in nanosized ferromagnets by spin injection. This technique promises to advance the speed of magnetic data recording into the picosecond time scale [4], while simplifying the process at the same time. In order 
to be able to thoroughly exploit this effect, precise understanding of the interaction between carrierspin and magnetic properties of the conducting material is necessary. Here, we present a new approach to determine electronic spin-precession in single crystalline magnetic films by SPLEEM. Using this method multiple perfectly well defined film thicknesses can be probed at once immediately allowing for a precise determination of the electronic precession angle per atomic layer. That way several different magnetic materials can be analyzed in a short period of time giving accurate, conclusive results which clearly demonstrate the success of this new approach to determine electronic spin precession by SPLEEM [5].

\section{References}

[1] R. Zdyb and E. Bauer, Phys. Rev. Lett. 88 (2002) 166403.

[2] D. Oberli et al., Phys. Rev. Lett. 81 (1998) 4228.

[3] W. Weber et al., Science 291 (2001) 1015.

[4] C.H. Back et al., Science 285 (1999) 864.

[5] This research was sponsored by the Austrian Science Fund (Schroedinger project J2352-N02).
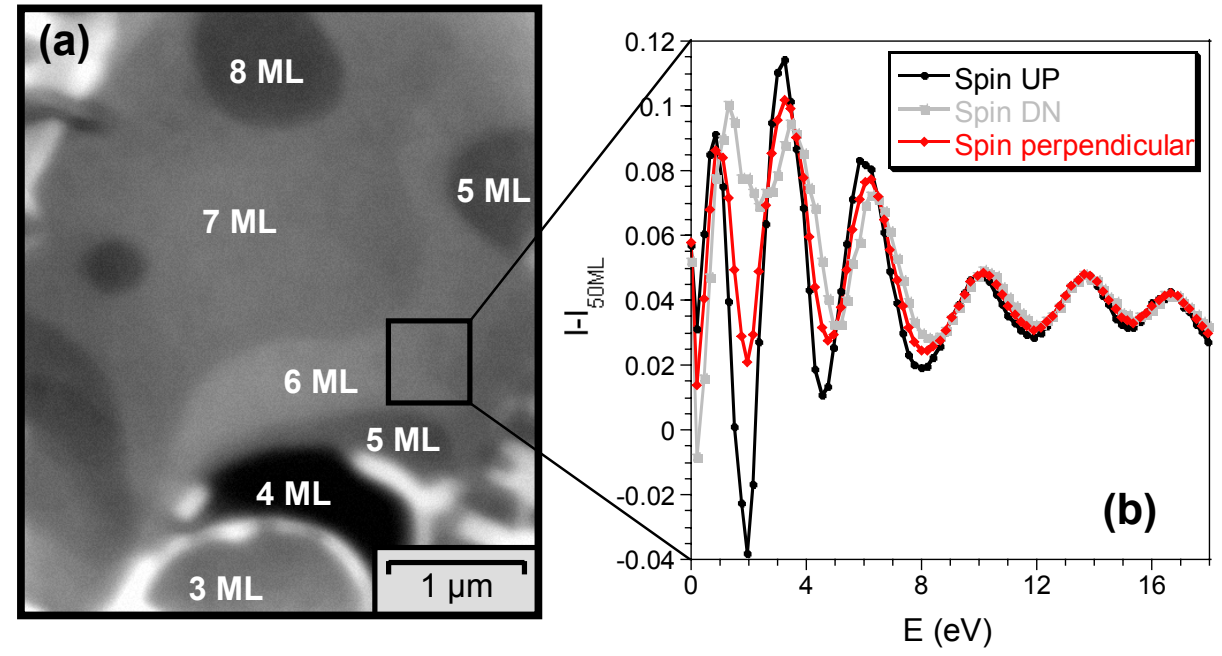

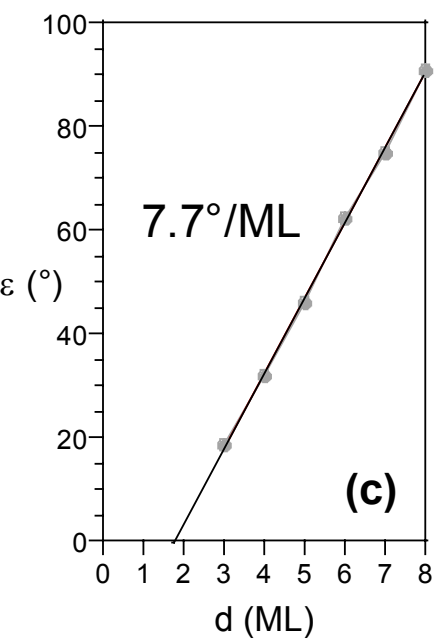

FIG. 1. (a) LEEM image of a large, atomically flat Co island grown on W(110) $\left(3.86 \times 4.4 \mu^{2}\right)$. Here, the topography of the underlying substrate is clearly visible since regions of different thickness show different reflectivity due to quantum size effects. (b) Energy-dependent mean reflectivity of a $6 \mathrm{ML}$ thick Co film, normalized by subtracting the reflectivity spectrum of a $50 \mathrm{ML}$ thick Co film (energy values given with respect to vacuum level). Spin UP/Spin DN curves show results for initial beam polarization parallel/anti-parallel to the magnetization of the sample. Information on the phase-shifts accumulated by travelling back and forth in the film can be extracted from the positions of the reflectivity maxima and minima. The energy-dependent relative phase-shift between UP and DN then determines the spin-precession angle. The result for a beam which is polarized perpendicular to the magnetization vector gives no new information since it is found to be exactly $(\mathrm{UP}+\mathrm{DN}) / 2$ (red diamonds). (c) Experimentally obtained precession angle plotted against Co-film thickness for electron energies of $10 \mathrm{eV}$ (above vacuum level) and 33\% initial beam polarization. The electronic spin-precession angle per atomic layer is given as half of the slope of the plotted linear regression line since every layer is passed through twice in our experiments. 